\title{
Effect of nanoparticles on spontaneous « Ouzo » emulsification
}

Clément Goubault ${ }^{1}$, Déborah Iglicki ${ }^{1}$, Robert A. Swain ${ }^{2}$, Benjamin F. P. McVey ${ }^{2}$, Bertrand Lefeuvre ${ }^{1}$, Ludivine Rault ${ }^{4}$, Céline Nayral ${ }^{2}$, Fabien Delpech ${ }^{2}$, Myrtil Kahn $^{3}$, Soizic Chevance ${ }^{1}$, and Fabienne Gauffre $^{1 *}$

${ }^{1}$ Univ Rennes, CNRS, ISCR-UMR6226, F-35000 Rennes, France.

${ }^{2}$ LPCNO, Université de Toulouse, CNRS, INSA, UPS, 135 Avenue de Rangueil, F-31077 Toulouse, France.

${ }^{3}$ LCC, CNRS, F-31000 Toulouse, France.

${ }^{4}$ Univ Rennes, CNRS, ScanMAT - UMS 2001, F-35000 Rennes, France

\begin{abstract}
Particles stabilize fluid interfaces. In particular, oil/water Pickering emulsions undergo limited coalescence, yielding droplets of smaller size as the amount of particles is increased. Herein, we studied the effect of hydrophobic nanoparticles ( $<10 \mathrm{~nm}$, alkyl-coated) on submicronic droplets ( $c a$ $100 \mathrm{~nm}$ ) formed in an Ouzo system. We investigated thoroughly the water/THF/BHT reference diagram, in the absence and in the presence of nanoparticles, using the Nanoparticle Tracking Analysis (NTA) technique. This allowed us to characterize the size distributions in a much finer way than what is usually obtained using conventional Dynamic Light Scattering (DLS). Both a Surfactant-Free Microemulsion (SFME, thermodynamically stable) and an Ouzo (metastable spontaneous emulsion) domains were identified and the transition from one to the other could be characterized by specific features of the droplet size distributions. We found that the presence of the nanoparticles limits coalescence in the metastable domain. We also show that the alkyl-coated nanoparticles are irreversibly attached to the liquid-liquid interface.
\end{abstract}

\section{Introduction}

A certain number of anis-flavored liqueurs traditionally consumed around the Mediterranean basin are drunk diluted in water. Examples are Pastis in France, Ouzo in Greece and Raki in Turkey. Adding a large excess of water to a premixed water/alcoholic solution containing aromatic oils causes the solution to turn milky. This phenomenon, known as the "Ouzo effect" since the seminal work of Vitale and Katz, ${ }^{1}$ is now partly understood: the ternary water/ethanol/oil system is abruptly driven into a metastable supersaturated state, located between the binodal (which marks the limit beyond which a homogeneous phase is most energetically favorable) and the spinodal (beyond which the homogeneous system can no 
longer exist and spontaneously separates into two phases). Supersaturation causes the nucleation and growth of submicronic oil-rich droplets that are very homogeneous in size and extremely stable.

Many synthetic Ouzo systems have been studied. They are composed of water, a water-miscible solvent such as ethanol, acetone, tetrahydrofuran (THF)... and a hydrophobic solute. ${ }^{1-5}$ The solute may be liquid or solid. Thus, the Ouzo effect is widely exploited in the pharmaceutical industry to coprecipitate drugs and polymers into nanoparticles of controlled size. The nanoprecipitation is driven by a "solventshifting" effect, i.e. adding an excess of a poor solvent (water) for the solute. ${ }^{6}$ Interestingly, when the solute is liquid, the Ouzo effect is a case of spontaneous emulsification (it requires neither energy nor surfactant). A narrow size distribution is obtained during the nucleation step, if the nucleation is fast and homogeneous, or, in other words, if mixing of water and solvent phases is faster than phase separation. ${ }^{6,7}$ Therefore, the most important control parameters are the kinetics of solvent diffusion in water and the conditions of supersaturation. ${ }^{1}$ The effect of composition, and most particularly of the degree of supersaturation, on the size of droplets produced via solvent exchange was clearly evidenced by flow experiments. ${ }^{89}$ Two phenomena may be responsible for the evolution on longer time scales: Ostwald ripening and coalescence. Ostwald ripening results from the exchange of molecules from the smaller droplets to the larger ones, due to the difference in Laplace pressure. Therefore, it is much slower when size dispersity is low. On the other hand, Ouzo emulsions are generally very dilute: the low concentration of droplets limits their coalescence. In addition, interfaces are generally electrically charged in water, which induces repulsive energy barriers between droplets. Botet and $\operatorname{Roger}^{7}$ calculated the interaction potential between two droplets of different sizes, considering both van der Waals attraction and electrostatic forces within the framework of DLVO theory. Their calculations evidence that the energy barrier is lower when the sizes of the two droplets are very different. This induces a narrowing of the size distribution with time. Finally, depending on their composition and conditions of preparation, the spontaneous emulsions formed by solvent shifting may exhibit a narrow size distribution and be stable over relatively long period of times (days or more). The Ouzo domain is generally defined as the region in the metastable domain, where submicronic particles of low size dispersity are obtained. ${ }^{2,6}$ However some authors define both a "stable" Ouzo domain (particles of homogeneous size) and an "unstable" Ouzo domain (polydisperse particles). ${ }^{4}$ It is important to note that the limits of the Ouzo domains and the final morphology of the particles may vary depending on the formulation pathway and the mixing method, since it correspond to out-of-equilibrium states. ${ }^{4}$ Therefore, the terminology "composition map" may be preferred to "phase diagram".

In addition to the metastable domain, many ternary systems made of water, a water-miscible solvent and an oil form surfactant-free microemulsions (SFME) in their monophasic domain. ${ }^{10}$ SFME were first evidenced in the late seventies by Barden and coworkers, using centrifugation and conductivity measurements. ${ }^{11}$ More recently, Anisimov and co-workers demonstrated that tert-butyl alcohol/water mixtures form stable aggregates of $\sim 100 \mathrm{~nm}$ in the presence of traces of propylene oxide. These are 
made of a solvent-swollen propylene oxide core surrounded by a mixed corona of tert-butyl alcohol and water. ${ }^{12}$ Similarly, Sedlák and co-workers found that THF/water mixtures also contain mesoscale inhomogeneities, due to the presence of hydrophobic impurities such as butylated hydroxytoluene (BHT, used as stabilizer) in THF. ${ }^{13-15}$ The reason for the formation of SFME is still unclear, however the role of the amphipatic-solvent (i.e. partially miscible with water and the oil) is central. In contrast to Ouzo emulsions, SFME are thermodynamically stable and optically transparent. Note that SFME with sizes as small as a few nanometers were also described. ${ }^{16}$ For a comprehensive review of SFME see Hou et $\mathrm{al}^{10}$.

In the same way as conventional water/oil emulsions, the Ouzo and SFME domains can be used to template the formation of materials. However, while conventional emulsions are usually in the range 1 $\mu \mathrm{m}-1 \mathrm{~mm},{ }^{17}$ Ouzo systems offer the possibility to reach the submicronic scale. Thus, submicronic polymer capsules were synthesized by co-precipitation of polymers in either the SFME or Ouzo domains of the water/acetone/miglyol (or hexadecane) system. ${ }^{3,18}$ With the objective to create capsules of inorganic nanoparticles (NP), we recently introduced NP into an Ouzo system. These NP were hydrophobic and of typical size $5-10 \mathrm{~nm}$. We demonstrated that the NP adsorb at the surface of submicronic droplets of diameter $\sim 100 \mathrm{~nm}$. The so formed NP-shells could be stabilized by a crosslinking polymer, forming capsules called Hybridosomes ${ }^{\circledR}$ with outstanding mechanical stability. ${ }^{19,20}$ TEM analysis showed that the wall of the capsules is made of a monolayer of NP. ${ }^{20}$ In addition, thanks to the specific process based on nanoprecipitation, they can encapsulate a high load of nanoprecipitated drugs, through a one-pot process. ${ }^{21}$

Particle-stabilized interfaces, whether in the case of Pickering emulsions or armored bubbles, show generally higher mechanical strength and longer time stability than surfactant-stabilized systems. This is due to the fact that the adhesion energy of particles, even as small as a few nanometers, is orders of magnitude larger than that of molecular surfactants. This characteristic is advantageous for encapsulation applications, where long-term stability may provide a substantial benefit. It also enables their use for the elaboration of advanced materials, such as solid foams and capsules. ${ }^{17,22}$

Here, our objective is to study the effect of NP on an Ouzo system, which until now had not been investigated. The water/THF/BHT system has emerged in our previous studies as a reference system for the development of NP-shells and Hybridosomes ${ }^{\circledR},{ }^{19-21}$ as THF is one of the few water-miscible solvents capable of both forming an Ouzo system with water and dispersing hydrophobic alkyl-coated NP. In addition, BHT is frequently present in commercial THF as a stabilizer to prevent radical polymerization of THF. In addition to DLS measurements, we have taken advantage of the Nanoparticle Tracking Analysis (NTA) ${ }^{23}$ technique to characterize the size distribution of droplets. NTA can determine the size distribution of suspensions of particles in the range 20-1000 $\mathrm{nm}$. Even if particles in this range are too small to be directly observed using optical microscopy, when they are shined by a laser beam, they each 
generate a diffraction spot clearly visible under a microscope. The Brownian motion of each of these spots is analyzed to determine the diffusion coefficient of each associated particle (or droplet in our case). Therefore, this technique enables to track the trajectory of individual nanoparticles and allows a straightforward measurement of their individual hydrodynamic diameter. It follows that NTA yields directly number-weighted size distributions, whereas the "natural" output of DLS is intensity-weighted size distributions which are less intuitive to interpret. ${ }^{24}$ Importantly, the concentration of particles can also be estimated by NTA.

In addition to DLS measurements, we have taken advantage of the Nanoparticle Tracking Analysis (NTA) technique to characterize the size distribution of droplets. Indeed, NTA is a microscopy technique enabling to track individual nanoparticles and allowing a straightforward measurement the diffusion coefficient for each particle from the analysis of their Brownian motion. As a consequence, NTA yields directly number-weighted size distributions, whereas the "natural" output of DLS is intensity-weighted size distributions which are less intuitive to interpret. Importantly, the concentration of particles can also be estimated by NTA. We have carried out a comprehensive study of the water/THF/BHT phase diagram in the absence of NP, highlighting both metastable and SFME domains. Then, we studied the influence of NP on both the metastable and SFME domains. Two types of NP were investigated, one type with ligands strongly anchored to the surface and the other with fluxional ligands, i.e. in dynamic exchange with the solution. These latter may modify the interfacial tensions. Finally, we discuss the fundamental similarities and differences with Pickering emulsions.

\section{Results}

Water/THF/BHT composition map. In order to have a reference diagram, we constructed the water/THF/BHT composition map (Figure 1). This was achieved using a BHT-free THF. Each point in the map corresponds to a sample prepared by adding water -in one shot- to a solution of BHT in THF. The sample was then rapidly vortexed to ensure homogeneity of the mixture. Following the suggestion of Vitale and Katz, ${ }^{1}$ the composition map is represented in an orthogonal basis with the solute (BHT) mass fraction along the $\mathrm{x}$-axis and the solvent (THF) mass fraction along the $\mathrm{y}$-axis. The water content is obtained by difference. A logarithmic scale was used to represent the concentration of BHT, which is much lower than that of the other two components. 


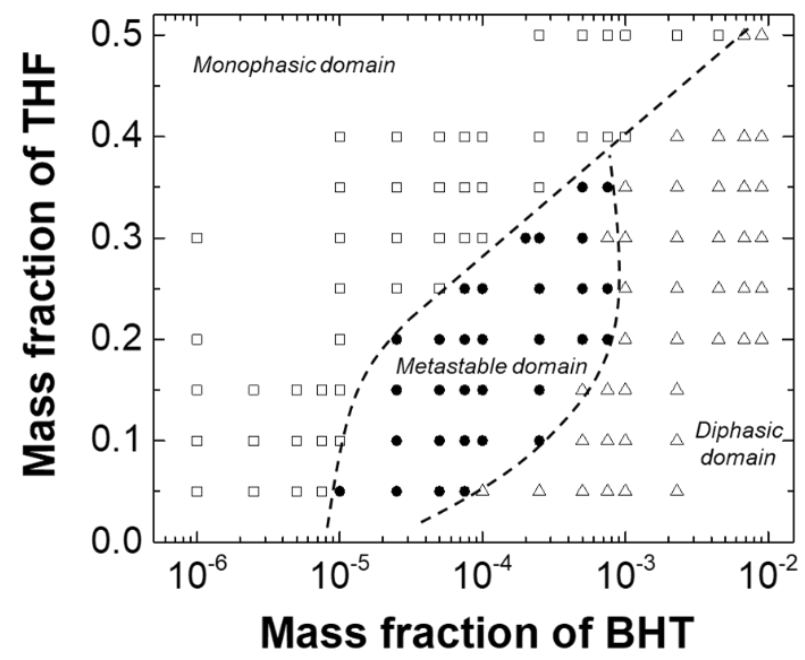

Figure 1 : Composition map of the water/THF/BHT system. Three domains were determined from visual inspection of the samples: $(\square)$ limpid and homogeneous samples (monophasic domain); $(\bullet)$ turbid samples (metastable domain); ( $\Delta$ ) biphasic samples. Dotted lines are guides for the eye.

Three domains can be distinguished based on visual observation of the samples. (i) A single-phase domain (empty squares), at low BHT concentration, where the samples are characterized by a transparent homogeneous aspect. (ii) An intermediate metastable domain (black dots), where the samples are turbid. (iii) Finally, at high BHT concentration, a domain in which demixed samples are observed (empty triangles). These latter samples are characterized by a few oily droplets that deposit on the walls of the vial when the sample is tilted.

Beyond simple visual observation, NTA allowed us to characterize the transition between single-phase and metastable domains in a more objective way. Indeed, NTA provides fine size distributions as well as an estimation of the dispersed phase concentration. Figure 2(a-c) shows the evolution of the histograms measured by NTA when increasing the concentration of BHT, along the lines $\Phi_{\mathrm{THF}}=0.1$; 0.2 and 0.3 (weight fractions). Three regimes can be distinguished: (i) at very low BHT fractions, the distributions show a main mode whose size does not vary significantly with BHT concentration $\left(\Phi_{\mathrm{THF}}=0.1\right.$ shows an additional mode superimposed on the main one); (ii) at intermediate BHT concentrations, this main mode shifts to larger sizes with increasing BHT concentration; (iii) finally, at large BHT concentrations the size distribution suddenly broadens and no main mode can be longer identified. The hydrodynamic diameters corresponding to the main mode in the first two regimes are reported (Figure 2d), as a function of the mass fraction of BHT. The presence of a well-defined monomodal peak in samples outside the Ouzo domain may seem surprising at first sight, but is probably the signature of a SFME. ${ }^{10,16,25}$ Note that the SFME domain extends at least down to $\Phi_{\mathrm{BHT}}=10^{-6}$ for the three THF fractions investigated. The measured main modes at this BHT concentration are in between 50 and $70 \mathrm{~nm}$. The increase in droplets size with increasing BHT concentration in the second regime is 
consistent with previous works reporting that in the Ouzo domain, increasing the oil content causes the droplet size to increase. ${ }^{1,2}$

Interestingly, the NTA also provides an estimation of the concentration of particles forming the dispersed phase. The three series of histograms (Fig2 a-c) exhibit one high intensity mode at a specific fraction of BHT $\left(\Phi_{\mathrm{BHT}}=10^{-5}\right.$ for $\Phi_{\mathrm{THF}}=0.1$ and 0.2 ; and $\Phi_{\mathrm{BHT}}=5 \times 10^{-5}$ for and $\left.\Phi_{\mathrm{THF}}=0.3\right)$. Currently, we do not have an explanation for this maximum concentration of droplets. However, these compositions seem to correspond to the limit between limpid and turbid samples as visually observed. In addition, they also mark the onset of the steep increase in droplet sizes (Figure 2d). Therefore, these specific compositions with a high concentration of droplets and low size dispersity may indicate the boundary between monophasic and Ouzo domains. Videos of NTA recordings are displayed in the Supporting Information for three representative samples along $\Phi_{\mathrm{THF}}=0.2$ : sample in the SFME domain $\left(\Phi_{\mathrm{BHT}}=10^{-6}\right.$; Video SI-1); monodisperse sample at maximum concentration of droplets $\left(\Phi_{\mathrm{BHT}}=10^{-5}\right.$; Video SI-2); polydisperse sample in the metastable domain $\left(\Phi_{\mathrm{BHT}}=10^{-4}\right.$; Video SI-3).
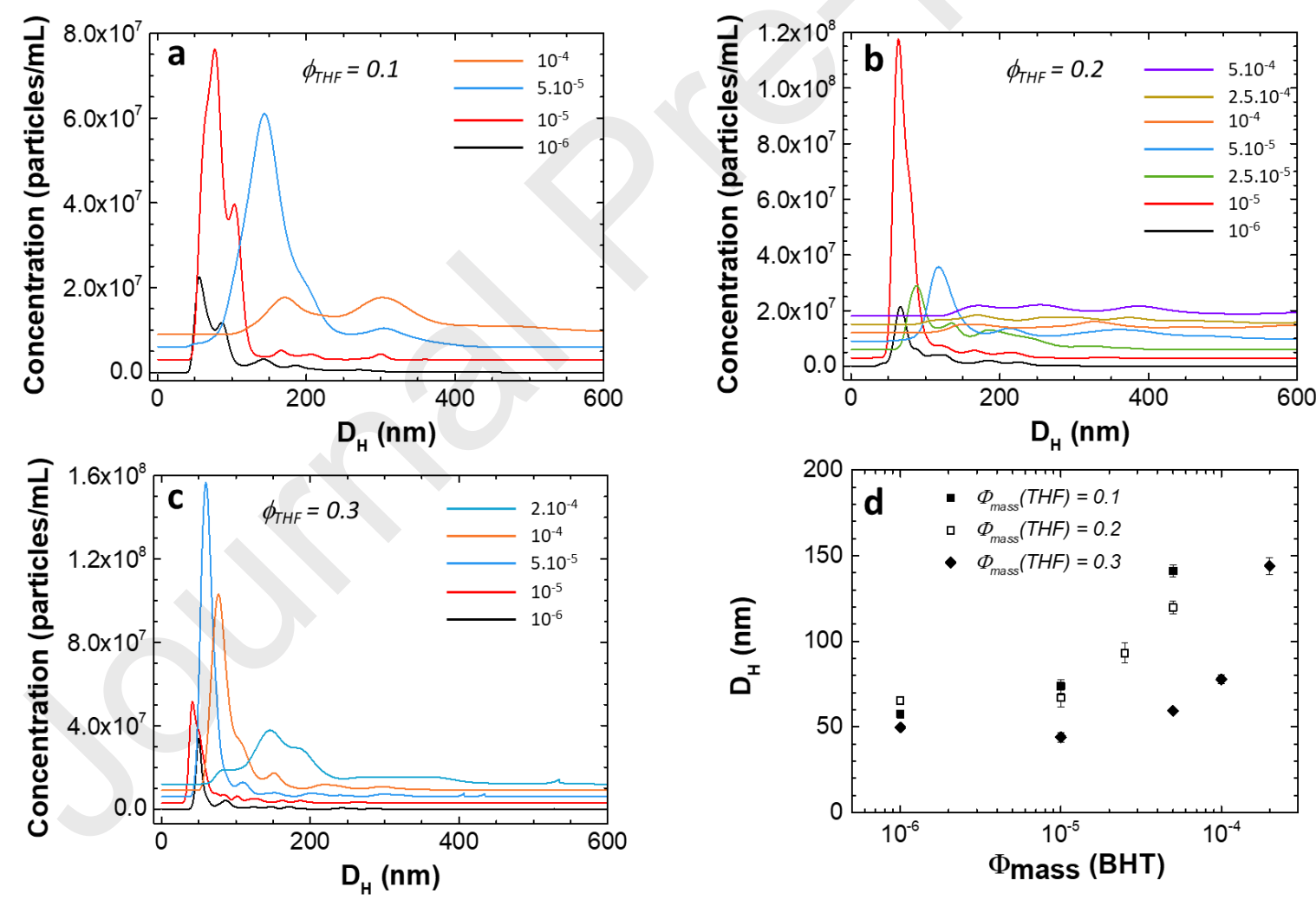

Figure $2:(a-c)$ Histograms of the hydrodynamic diameters measured using NTA for water/THF/BHT mixtures with increasing mass fraction of BHT (indicated on graphs), at fixed THF contents $\left(a: \Phi_{T H F}=0.1 ; b: \quad \Phi_{T H F}=0.2 ; c: \Phi_{T H F}=0.3\right)$. (d) Hydrodynamic diameters of the main mode (for the monomodal distributions) as a function of increasing fraction of BHT. 
Effect of nanoparticles on the Ouzo system. The main objective of this work is to investigate the effect of hydrophobic NP on the water/THF/BHT system. As model particles, we first selected commercial Quantum Dot nanocrystals (Cytodiagnostics) with a core $\left(\mathrm{Cd}_{\mathrm{x}} \mathrm{Se}_{1-\mathrm{x}}\right) /$ shell $(\mathrm{ZnS})$ structure and stabilized with oleic acid. Oleate is known to anchor strongly to the surface of these QD. The QD (initially in toluene) were purified before use by centrifugation to remove any excess ligand and dispersed into THF. Characterization by high-resolution transmission electron microscopy (HR-TEM) shows a nanocrystalline structure with a faceted shape and a mean size of 7.8 (1.2) nm (Figure SI-1) (figures in brackets represent the standard deviation).

First, we examined samples along a dilution line (dilution of a THF/BHT solution with water) at constant particle concentration (estimated at $3 \times 10^{13} \mathrm{QD} / \mathrm{mL}$ from supplier information). Five samples were prepared with water mass fractions of $0.11 ; 0.32 ; 0.53 ; 0.67$ and 0.77 . The location of the samples on the composition maps and photographs of the samples after 4 days are shown Figure SI-5. The QD precipitate for the four samples with water mass fraction $<0.7$, leaving a clear supernatant. In contrast, the sample at $\Phi_{\text {water }}=0.77$ remains turbid, but shows no sign of precipitation. The precipitation of the QD's in the presence of water, even in proportion as low as 0.11 is expected since these particles are grafted with hydrophobic alkyl chains. More surprising is the stability of the sample at $\Phi_{\text {water }}=0.77$, which lies in the metastable domain. This unexpected stability of hydrophobic NP at high water content is a general phenomenon which was previously reported by us in the case of gold and iron oxide NP. ${ }^{19}$

In a second step, we investigated the influence of NP on the horizontal line corresponding to $\Phi_{\mathrm{THF}}=0.2$ of the composition map. The visual comparison of the samples without NP or containing $1.5 \times 10^{13}$ $\mathrm{NP} / \mathrm{mL}$ shows that the transition between the monophasic and the metastable domain is unchanged in the presence of NP. Figure 3 enables the comparison of the size histogram of two compositions in the metastable domain in the absence and in the presence of NP $(10 \mu \mathrm{g} / \mathrm{mL})$. Very interestingly, in the presence of NP, the histograms (Figure 3b 1\&2) show a well-defined main mode centered at 139 and $150 \mathrm{~nm}$ (for $\Phi_{\mathrm{BHT}}=10^{-4}$ and $\Phi_{\mathrm{BHT}}=2.5 \times 10^{-4}$ respectively), whereas the corresponding compositions without NP show a broad distribution spanning over the entire measurement range (Figure 3 a $1 \& 2$ ). Thus, the presence of NP has a strong influence on the size distribution, with the effect of narrowing the size distribution. The comparison of two series of samples -with and without NP- with increasing concentrations of BHT are represented in Figure 3c\&d. Regarding the size (Figure 3c), the two sets of data show the same trend at low BHT fractions. However, the broadening of the distribution is not observed in the presence of NP, whereas it occurs from $\Phi_{\mathrm{BHT}}=10^{-4}$ when they are not present. Regarding the concentration of the droplets, it appears that the droplets are much more numerous in the presence of NP, especially at low BHT fractions. The NTA video acquisitions corresponding to Figure 3 a1 and b1 are provided in the Supporting Information (Video SI-a1 and b1) and clearly show this increase in concentration. 
To summarize, these results show that the single-phase to metastable domain transition is not shifted in the presence of NP. However, the Ouzo domain (showing a narrow distribution of droplets) is extended towards larger solute concentrations.
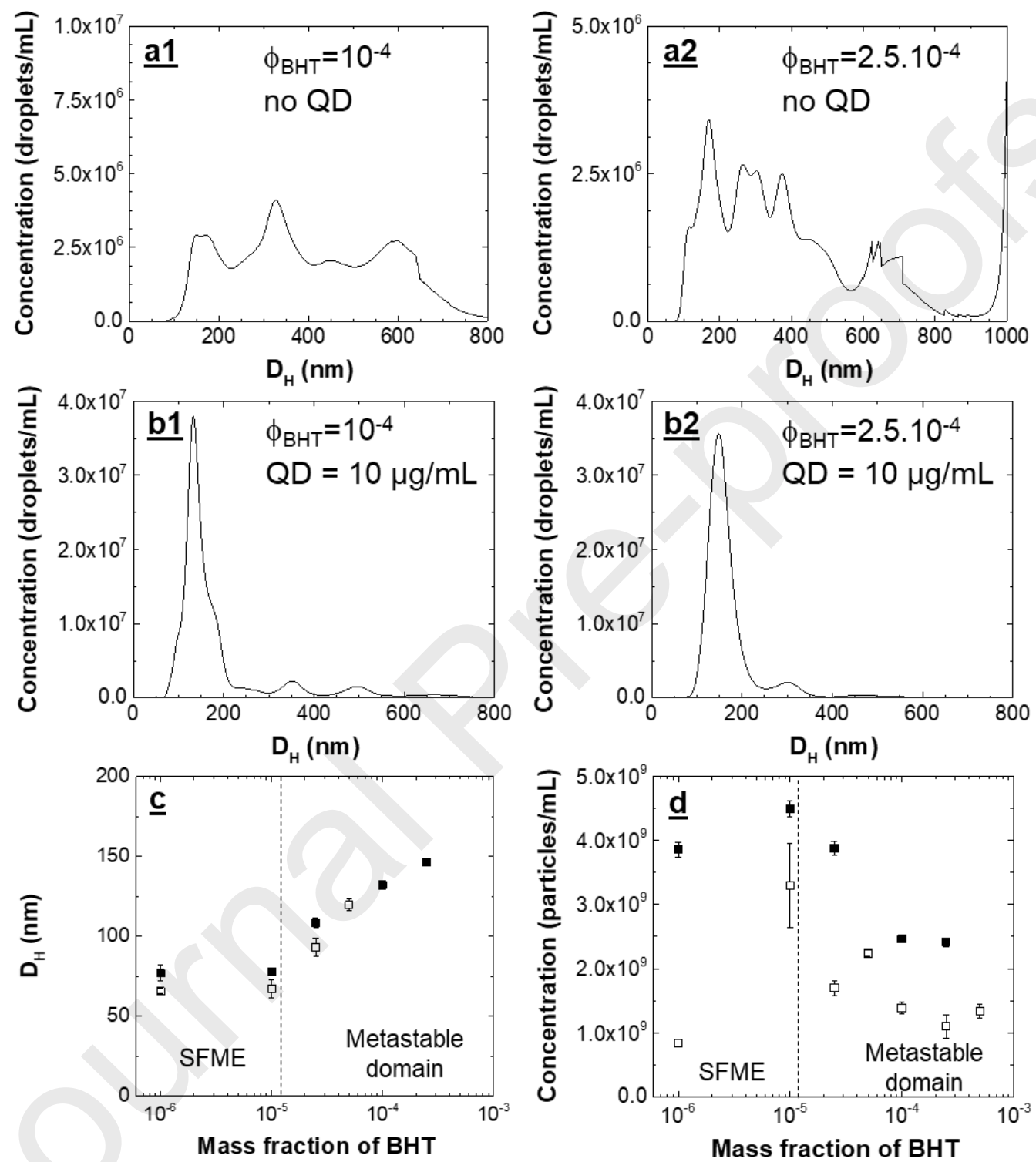

Figure 3 : Comparison of the size distribution measured by NTA in the absence (a1-a2) and in the presence (b1-b2) of QD for two given compositions (a1-b1: $\left.\Phi_{T H F}=0.2 ; \Phi_{B H T}=10^{-4}\right)$ and $\left(a 2-b 2: \Phi_{T H F}=0.2 ; \Phi_{B H T}=2.5 \times 10^{-4}\right)$ in the metastable domain. (c) Size (main mode of the distribution) and $(d)$ concentration of the droplets, in the absence $(\square)$ and in the presence $(\mathbf{\square})$ of the $Q D(10 \mu \mathrm{g} / \mathrm{mL})$. The size histograms al and a2 show no principal mode, and are not represented on figure c. All compositions stand along the line $\Phi_{T H F}=0.2$.

Effect of the nanoparticle concentration on droplet growth. Previous works have shown that, in the Ouzo domain, the droplets form by a mechanism of nucleation and growth as described by the theory of 
La Mer. ${ }^{1,2,7}$ The rapid addition of water abruptly drives the system beyond the solubility limit of the oil (here BHT) and forces it to "precipitate", i.e. nucleate droplets. These droplets grow rapidly, fed by the excess oil, until concentration in the continuous phase reaches the limit of solubility. In a second step, the droplets grow through a coalescence mechanism. In the case of oil/water Pickering emulsions, it is known that the presence of particles induces a phenomenon of limited coalescence, which results in a slower coalescence rate and eventually smaller droplets. ${ }^{26}$ In addition, limited coalescence yields a characteristic proportionality law relating the inverse of the droplet size to the amount of particles.

We measured the size of the droplets in samples containing various amounts of QD in the range 0-40 $\mu \mathrm{g} / \mathrm{mL}$, three hours after their preparation (Figure 4). The droplet sizes obtained via DLS analysis of the samples are shown in Figure 4a. With the exception of very low NP concentrations (not represented), the droplet size shrinks steadily with increasing the QD concentration. Figure $4 \mathrm{~b}$ shows the inverse of the size as a function of the QD concentration, evidencing a linear relationship, which could be due to limited coalescence. However, the intercept of the line is not 0 , as observed for Pickering emulsions. Indeed, the Ouzo emulsions already show long-term stability in the absence of NP. Here the size of the droplets in the absence of NP was measured at $D_{h}=195 \mathrm{~nm}$. As mentioned earlier, this is thought to result from the spontaneous charge of the liquid-liquid interface in water. The presence of a charged interface was confirmed by zeta potential measurements in the absence of NP (-23.5 (4) mV).
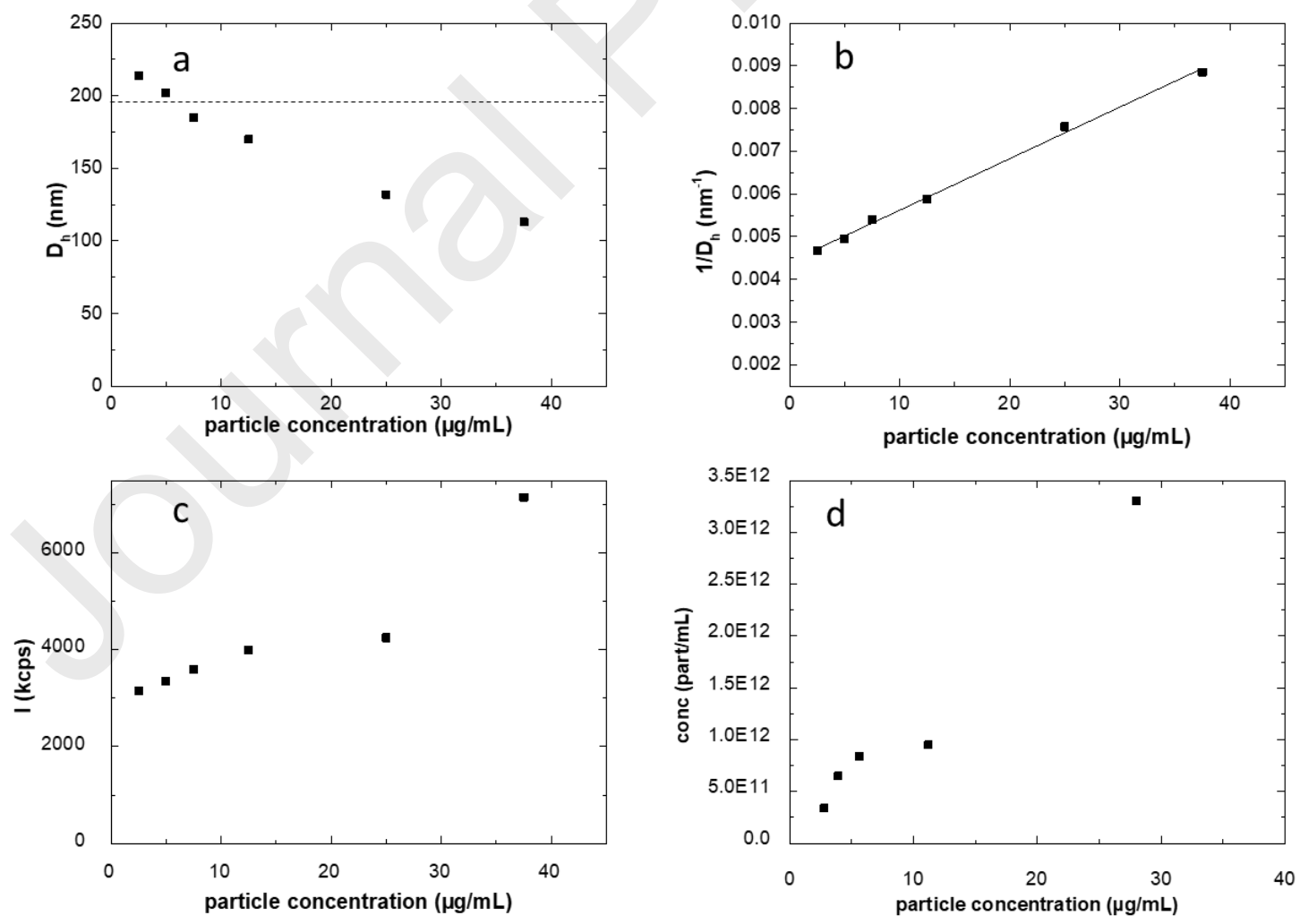
Figure 4 : Influence of the amount of $Q D$ on the characteristics of the droplets in the Ouzo domain, from DLS (a-c) and NTA measurements $(d)$. (a) Hydrodynamic diameter. The dotted line represents the size measured at $[Q D]=0 ;(b)$ Inverse of the diameter. The full line represents the best linear fit; $(c)$ mean scattered intensity; $(d)$ concentration determined by NTA after crosslinking of the NP-shell. For all samples: $\Phi_{T H F}=0.23 ; \Phi_{B H T}=7.3 \times 10^{-5}$.

We could not determine the concentration of droplets by NTA for the samples at high QD concentration, due to fluorescence and concentration issues. Therefore, we used another strategy based on our previous work. ${ }^{19,20}$ The QD-shells were cross-linked using a poly(acrylic acid) prior to dilution. After centrifugation in order to remove excess polymer, the residual solvent was evaporated and all samples were diluted with an appropriate amount of water to enable a reliable determination of the concentration using NTA (between 2 and $8.5 \times 10^{8}$ particles $/ \mathrm{mL}$ ). Figure $4 \mathrm{~d}$ shows that the concentration of crosslinked NP-shells increases when more QD are present, indicating an increasing population of droplets. Note that the increase in the mean scattered intensity measured by DLS directly in the water/THF/BHT/QD mixtures (Figure 4c) is also a good indication of an increase in the number of droplets with the QD concentration. Indeed, the intensity scales as I $\sim \mathrm{N} \mathrm{D}^{6}$, where $\mathrm{N}$ and $\mathrm{D}$ stand respectively for the number and the radius of the scatterers. Since the size of the droplets decreases (Figure 4a), the continuous increase of in intensity can only be explained by a multiplication of the number of droplets. To summarize, two trends are clearly observed when the QD concentration is increased: a decrease in the size of the drops and an increase in their number.

We also monitored the dynamics of droplet growth using Dynamic Light Scattering (DLS). Thanks to the specific correlator of the instrument used (Nanokin, Cordouan), we could record the scattered intensity continuously throughout the experiment, starting approximately 10 seconds after mixing. Then, the auto-correlated function was calculated from regularly distributed time sequences of the scattered intensity. The dynamics at short timescales $(<10 \mathrm{~s})$ was not accessible by DLS because it takes a few seconds for the system to homogenize microscopically and another few seconds of measurement for the statistics of the auto-correlation signal to be satisfactory. The evolution of the droplets hydrodynamic radius over time is shown in Figure SI-6. Although it seems that the droplet size grows slower in the presence of increasing amount of NP, the early times of the kinetics are missing to determine the characteristic times of the kinetics quantitatively. Nevertheless, a plateau is reached after $c a 15$ minutes in all cases.

In an effort to generalize our findings, we selected two other types of NP: InP/ZnS core/shell nanocrystals and $\mathrm{Fe}_{2} \mathrm{O}_{3}$ (maghemite) nanocrystals (see SI-section A for characterization of these NP). These NP both measure ca $4 \mathrm{~nm}$, that is approximately half of the size of the CdSe/ZnS NP. Importantly, both of these particles are stabilized with fluxional ligands, i.e. partially exchanging with the solution. ${ }^{27,28}$ As previously observed, the inverse of the droplet size shows a linear relationship with the amount of NP (Figure 5). 

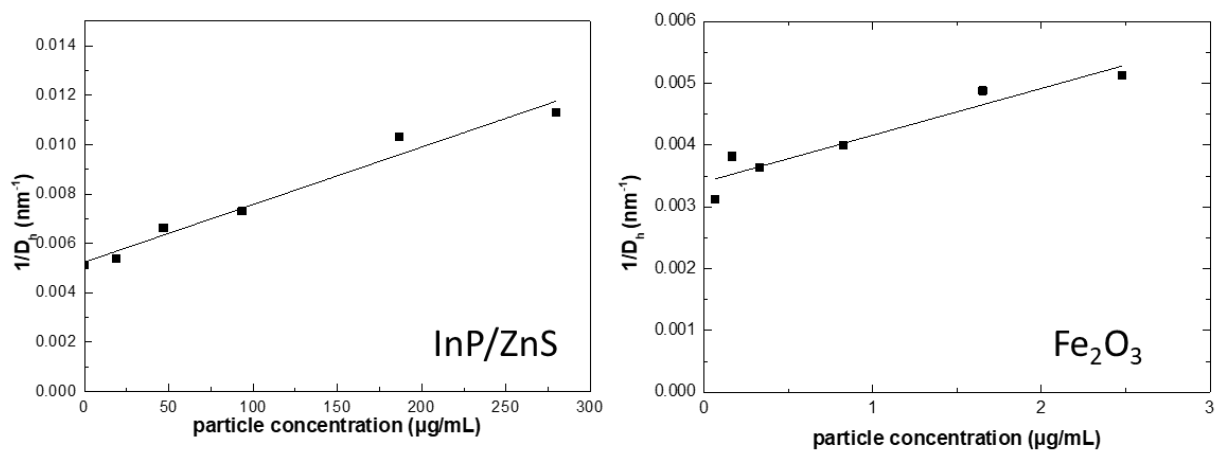

Figure 5: Inverse of the hydrodynamic diameter vs the amount of NP in the systems, for the two types of NP with fluxional ligands. Left: InP/ZnS nanocrystals; right: $\mathrm{Fe}_{2} \mathrm{O}_{3}$ nanocrystals.

Can the NP reversibly desorb/absorb from the interface? Finally, we investigated whether the NP at the liquid-liquid interface are irreversibly anchored or in dynamic exchange with the solution. To address this issue, we set up the following procedure. Two suspensions of NP-shells were first prepared separately with two different types of $\mathrm{NP}\left(\mathrm{CdSe} / \mathrm{ZnS}\right.$ or superparamagnetic $\left.\mathrm{Fe}_{2} \mathrm{O}_{3}\right)$. Then, they were mixed together and allowed to rest for NP exchange to occur. Finally, we looked for the presence of NPshells of mixed composition using either Energy Dispersive X-Ray Spectroscopy (EDX) or elemental analysis. The advantage of using $\mathrm{Fe}_{2} \mathrm{O}_{3} \mathrm{NP}$ is that their NP-shell can be separated from the suspension magnetically. As previously, NP-shells were crosslinked using PAA, and the solvent was evaporated before separation. We define the mixing time as the time between the mixing of the two NP-shell samples and the addition of the crosslinking polymer. The two fractions were investigated using transmission electron microscopy (TEM) and EDX. The pellet was used as such but the supernatant was centrifugated to remove excess polymer and free ions before TEM analysis. Figure 6 shows TEM images and typical EDX spectra of the magnetic and non-magnetic fractions for a mixing time of 1 hour. 

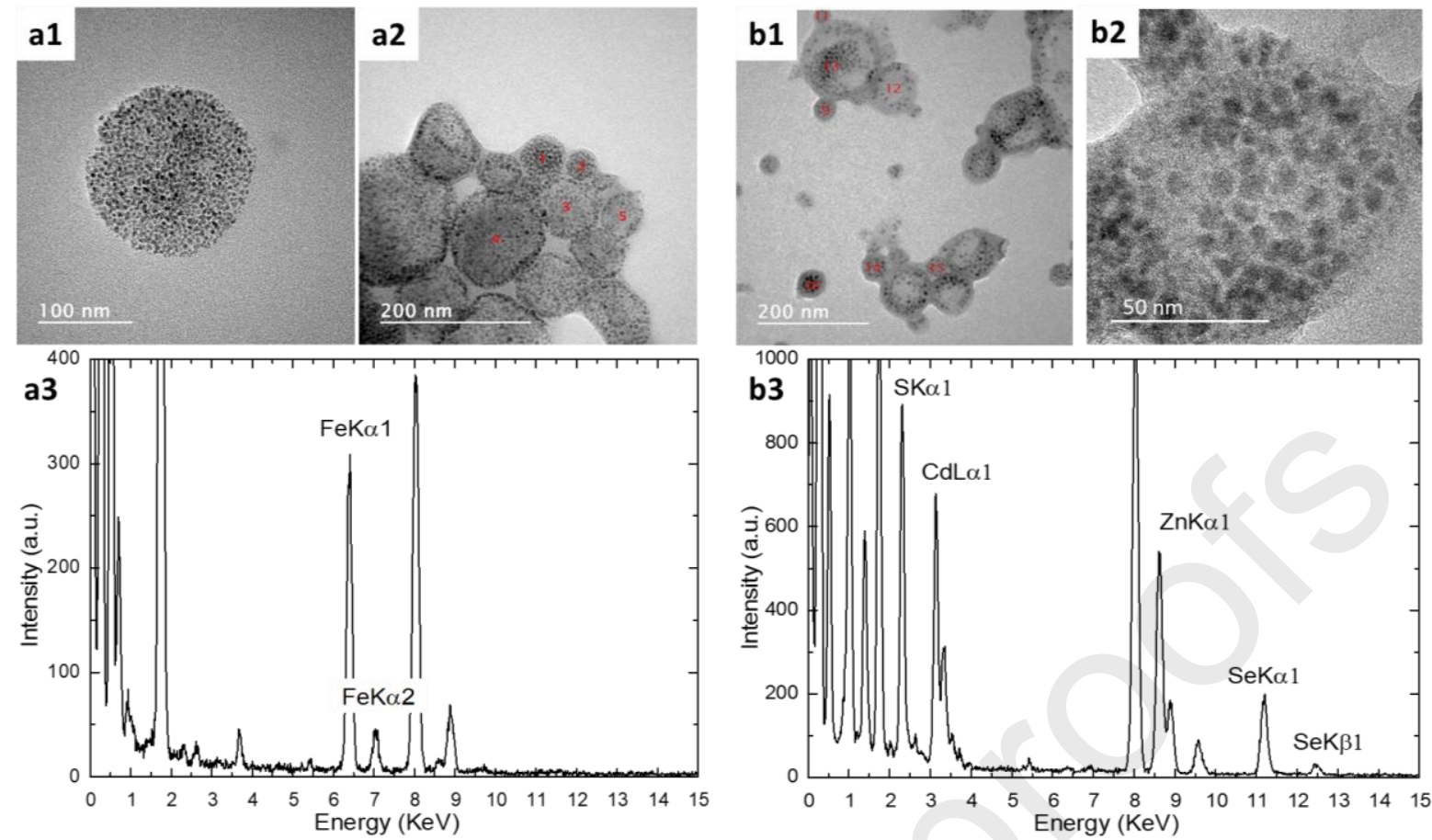

Figure 6 : TEM and EDX analysis of a mixture of $\mathrm{Fe}_{2} \mathrm{O}_{3}$-shells and $Q D$-shells after magnetic separation. (a) magnetic fraction; (b) non-magnetic fraction. NP-shells prepared from the composition: $\Phi_{T H F}=0.23 ; \Phi_{B H T}=8.2 \times 10^{-5}$ and $[Q D]=50 \mu \mathrm{g} / \mathrm{mL}$ or $\left[\mathrm{Fe}_{2} \mathrm{O}_{3}\right]=2.5 \mu \mathrm{g} / \mathrm{mL}$; Mixing time: 1 hour. EDX: a3=zone 3; b3=zone 13.

NP-shells are present both in the magnetic (Figure 6a) and non-magnetic (Figure 6b) fractions. The TEM contrast in the non-magnetic fraction is less pronounced and some parts of the NP-shells seem free of NP. However, a higher density of NP is observed using HR-TEM. Figure 6-a3 and b3 display typical EDX spectra of the magnetic and non-magnetic fractions respectively. The magnetic fraction contains iron but no cadmium or selenium. In contrast, the EDX spectra representative of the non-magnetic fraction shows the presence of $\mathrm{Cd}$ and Se but the absence of iron. It has to be noted however, that a few NP-shells observed in the non-magnetic fractions were made of iron only (no QD). This concerned only small capsules (typically $<40 \mathrm{~nm}$ ). These first results suggest that mixing does not occur, or on a much longer time scale. However, such microscopic measurements do not have a strong statistical value. Therefore, we also quantified the amount of $\mathrm{Fe}_{2} \mathrm{O}_{3}$ and QD in each fraction using elemental titration (ICP-AES). In this case, the elements $\mathrm{Fe}$ and $\mathrm{Cd}$ were titrated in the whole magnetic and non-magnetic fractions. In addition, the mixing time was increased to 5 hours. Elemental titration (Figure SI-7) shows that almost all QD's (94\%) are found in the non-magnetic fraction. While the majority of iron (73\%) is distributed in the magnetic fraction. We note that iron is also present in the non-magnetic fraction in a non-negligible amount (27\%). A similar repartition of iron is found for suspensions of pure $\mathrm{Fe}_{2} \mathrm{O}_{3}$-shells after magnetic separation. This is possibly due to NP-shell of smaller sizes and showing a weak magnetic moment. In conclusion, both the electron microscopy and elemental titration show that the NP are irreversibly adsorbed at the droplets surface. 


\section{Discussion}

Mechanisms that are possibly affected by the presence of the NP. The first objective of this discussion is to identify the possible mechanisms which are affected by the presence of NP. As already explained in the introduction, the Ouzo domain corresponds to a small region of the metastable region, where droplets of uniform size are formed. As a consequence of this narrow size distribution, Ostwald ripening and coalescence are not observed. This can be observed at low solute concentration, i.e. in the vicinity of the monophasic domain. When the solute concentration increases, the concentration of droplets increases causing coalescence and broadening of the size dispersity. Polydispersity can also result from a non-homogeneous nucleation due to nucleation occurring rapidly. These trends are clearly observed in the NTA size histogram displayed in Figure 2. Indeed, we locate the SFME to Ouzo transition where the distribution shows the most intense monomodal peak. These compositions also mark the onset of turbidity of the samples. From this boundary, the compositions with increasing solute concentration exhibit some larger droplets and eventually high dispersity. It's interesting to note that the compositions that are most altered by the NP presence are the most concentrated ones (compare for instance Figure 3a1 and 3b1). This raises the following question: do NP affect the nucleation step, the growth by condensation or a subsequent coalescence or ripening process?

The three types of NP added to the system are coated with hydrophobic alkyl chains. ${ }^{28}$ It was observed here and in previous studies ${ }^{19}$ that hydrophobic NP precipitate out of the solution when very small amounts of water are added, for instance less than 5\%. In our process, the NP were initially suspended in THF with BHT. Under these conditions, they are also expected to precipitate upon addition of water. However, due to the relatively large size of NP (compared to BHT), their precipitation occurs at a slower rate. For comparison, the diffusion coefficient $(D)$ of molecules such as THF or BHT in water (calculated from the Stokes-Einstein equation) is of the order of $10^{-9} \mathrm{~m}^{2} \cdot \mathrm{s}^{-1}$ whereas that of $\sim 5 \mathrm{~nm}$ particles is $2.4 \times 10^{-11} \mathrm{~m}^{2} \cdot \mathrm{s}^{-1}$. This equivalently means that the mean diffusion length of the molecular species is one order of magnitude larger than that of a $5 \mathrm{~nm}$ particle, since $l=\sqrt{2 . D . t}$. Therefore, one can consider that the initial droplets are formed before the precipitation of the NP. From this simple calculation, we assume that NP do not affect the nucleation step. This is further confirmed by the observation that the onset of the metastable domain is not modified in their presence.

A mechanism of limited coalescence was evidenced and expertly characterized in the case of Pickering emulsions. ${ }^{26}$ It can be summarized as follows. The emulsions are obtained by mixing the water and oil phases together under intense shearing. If the amount of interface produced at this initial stage is larger than the surface that can be covered by the particles $\left(S_{p}\right)$, the droplets grow by coalescence. This causes the total surface to decrease, until it matches with $S_{p}$. In this situation, it's easy to show that the total 
amount of particle (total mass $m$ ) should evolve linearly with the inverse of the mean droplet diameter $(\bar{D}):^{26,29}$

$$
\frac{1}{\bar{D}}=\frac{m s}{6 V}
$$

With the specific surface of the NP $\left(\mathrm{m} . \mathrm{s}=\mathrm{S}_{\mathrm{p}}\right)$, and V (the volume of the dispersed phase). Hence, the specific surface of the particles can be calculated from the slope of the line.

In the present work, we examined the variation of 1/D with the amount of NP, for three types of NP. From our results, we believe that we are also in a case of limited coalescence. Indeed, in all cases we found a linear relationship. The specific surfaces calculated from the slopes of the best linear fits are $\mathrm{s}=57 \mathrm{~m}^{2} \cdot \mathrm{g}^{-1} ; 11 \mathrm{~m}^{2} \cdot \mathrm{g}^{-1}$ and $355 \mathrm{~m}^{2} \cdot \mathrm{g}^{-1}$ for CdSe/ZnS, InP/ZnS and $\mathrm{Fe}_{2} \mathrm{O}_{3} \mathrm{NP}$ respectively. Note that for the two core/shell particles, the weight concentrations used in Figure 5 comprises an unknown amount of ligand. In the case of the iron oxide NP, it is expressed as the weight of the particles only, calculated from ICP measurement. The measured value compares well with the estimated value of 229 $\mathrm{m}^{2} \cdot \mathrm{g}^{-1}$, calculated assuming a diameter of $4.8 \mathrm{~nm}$ and a density of $4.9 \mathrm{~g} / \mathrm{cm}^{3}$ for $\mathrm{Fe}_{2} \mathrm{O}_{3}$.

Some important differences with the case of Pickering emulsions need to be examined. Let's first consider the evolution of the emulsion over the long term. In the absence of a stabilizer, Pickering emulsions will eventually reach total demixing $(\bar{D} \rightarrow \infty)$. This translates into the fact that the y-ordinate should cross 0 . This is obviously not the case in our experiments, because the Ouzo emulsions exhibit a finite droplet size even in the absence of NP. As mentioned previously, the finite size of the droplets can be at least partially explained by the electrostatic charging of the interfaces in water. The charge of the interface was confirmed here by a significant negative zeta potential $(-23 \mathrm{mV})$. On the other hand, at elevated particle concentrations, Pickering emulsions will eventually enter in a regime where $S_{p}$ is larger than the oil/water interface initially generated, resulting in an excess of free particles. In our case, the droplets generate spontaneously and the initial surface is not defined, so we can expect to continue reducing droplet size even at very high particle concentration.

It is also important to note that the presence of NP at the liquid-liquid interface most certainly decreases the interfacial tension. This would lower the effect of Ostwald ripening and therefore limit particle growth as observed here. However, the Ostwald ripening of emulsions is also characterized by size focusing (the smaller droplets feed the larger ones), ${ }^{30}$ which is clearly not the case here in the absence of NP.

To summarize, our results show that the NP narrow the size distribution of the emulsion in the metastable domain, by limiting coalescence. In contrast, they have no or little effect on droplet nucleation.

Origin of the adhesion of the NP at the droplets surface. When particles are forced to precipitate out of the solution a competition should take place between particle-particle interaction and particle-droplet 
interactions. In the range of NP concentrations investigated here, we did not observe any precipitation that would be a signature of NP-NP aggregation. Indeed, NP-NP interactions are prevented by their alkyl chain ligand. An experimental evidence of this steric repulsive interaction is that they're easily redispersable after aggregation induced by centrifugation. Therefore, even a small energy of adhesion at the droplet surface may be enough to favor droplet coating rather than NP-NP aggregation. At this stage, it is interesting to note that Ganachaud et al. created polymer capsules templated by Ouzo droplets, using also a co-nanoprecipitation strategy. ${ }^{3,18}$ The major difference with the work reported here is that the polymer is initially water soluble. However, in appropriate conditions, they found that the polymer precipitate at the surface of the droplets.

The nature of the forces that promote particle adhesion at the droplet surface remains unclear. In the case of Pickering emulsions, particles better adsorb at the oil/water interface, if they are of intermediate hydrophoby. ${ }^{22,31}$ This is due to the fact that if both liquids can wet the particle, the contact angle is close to $90^{\circ}$. Since the adhesion energy writes:

$$
\Delta G=\pi R^{2} \gamma(1-\cos \theta)^{2}
$$

where $\gamma$ is the oil/water interfacial tension, it is maximal for a contact angle close to $90^{\circ}$. In such conditions, it is generally admitted that the particles are irreversibly attached to the interfaces, even for NP with radius of a few nanometers. ${ }^{31}$ As mentioned previously, the NP are alkyl-coated and should be embedded preferentially in the dispersed phase resulting in a rather low adhesion energy. However, our experiments show no sign of particles exchange when NP-shells made from different particles are mixed together, proving that this adhesion is irreversible. This might be attributed to the very low solubility of the particles in the continuous phase.

\section{Conclusion}

We investigated for the first time the effect of NP on the characteristics of spontaneous submicronic Ouzo emulsions. Thanks to Nanoparticle Tracking Analysis we could provide a reliable analysis of the size distributions and an estimation of the droplet concentrations in the water/THF/BHT system. Importantly, we evidenced a sharp maximum of droplet concentrations together with a monomodal distribution at the transition from the monophasic to the Ouzo domain. The single phase to metastable transition is not significantly shifted in the presence of NP. However, the Ouzo domain is significantly extended toward higher solute concentration in the presence of NP. Thus, compositions showing droplets of all sizes, may exhibit a monomodal distribution in the presence of NP. The inverse of the droplet size growths linearly with NP concentration, as in the case of Pickering oil/water emulsions, although droplets are several orders of magnitude smaller. These results can be explained by a phenomenon of limited coalescence due to the formation of a shell of NP at the liquid-liquid interface. 
We also evidenced that the NP are irreversibly adsorbed at the droplet surface. Very interestingly, these Ouzo emulsions "armoured" with NP can be turned into solid nanoparticle capsules for material applications.

Acknowlegments: FG thanks warmly Lazhar Benyayah, Christophe Chassenieux, François Ganachaud and Isabelle Cantat for fruitful discussions. We wish to thank the region Bretagne (ARED- 107/ NAPOLI), the LCC and the ANR (grant: 2020-CES06-OuzoFan) for Financial support. FD thanks ERC Advanced Grant (MONACAT 2015-694159) and EUR grant NanoX no. ANR-17-EURE-0009.

\section{Material and methods}

Materials: Tetrahydrofuran (THF, GPR Rectapur $\geq 99 \%$ stabilized) was purchased from VWR and used as received. Samples were made using MilliQ water $\left(18.2 \mathrm{M} \Omega . \mathrm{cm}^{-1}\right)$. The hydrophobic Cadmium Selenide/Zinc Sulfide (CdSe/ZnS) core/shell quantum dots stabilized with oleic acid (emission at $665 \mathrm{~nm}$ ) were purchased from Cytodiagnostic. They are provided as a $5 \mathrm{mg} / \mathrm{mL}$ solution in toluene, or ca $1.2 \times 10^{15}$ nanocrystals per $\mathrm{mg}$ (data from supplier).

Ttransfer of the commercial QD in THF. The QD were transferred from toluene to THF by precipitation. An equal volume of methanol is added to the toluene solution. After 30 minutes of resting, the mixture was centrifugated and the pellet was resuspended in THF with a dilution factor of 10 relative to the initial toluene solution, reaching a final concentration of $6 \times 10^{14}$ $\mathrm{NP} / \mathrm{mL}$ according to the to the supplier's information. Characterization of the NP is reported in the Supporting Information.

Synthesis of $\gamma$-Fe $\mathrm{Fe}_{2} \mathrm{O}_{3}$ nanoparticles. The iron oxide $\left(\gamma-\mathrm{Fe}_{2} \mathrm{O}_{3}\right) \mathrm{NP}$ were synthesized following a reported procedure with slight modifications. ${ }^{32}$ Briefly, the iron oxide NP are obtained via hydrolysis of an organometallic Fe(II) precursor in the presence of a stabilizing ligand (here 2 eq. of octylamine and 1 eq. of oleic acid, relative to $\mathrm{Fe}$ ), in the absence of any other solvent. Then, the SPION were resuspended in $8 \mathrm{~mL}$ of THF. The vial was set standing over a permanent magnet overnight and the pellet eliminated. The amount of iron in the solution after magnetic sorting of the NP was determined by ICP-AES $\left(330 \mathrm{mg} \cdot \mathrm{L}^{-1}\right)$. Characterization of the NP is reported in the Supporting Information. 
Synthesis of InP/ZnS nanoparticles. In the glovebox, $161.6 \mathrm{mg}(0.3 \mathrm{mmol}) \operatorname{In}(\mathrm{amid})_{3}, 307.7$ $\mathrm{mg}(1.2 \mathrm{mmol})$ palmitic acid (PA), and $37.1 \mathrm{mg}(0.16 \mathrm{mmol})(\mathrm{TMS})_{3} \mathrm{P}$ were dissolved in $5 \mathrm{~mL}$ of mesitylene in a $100 \mathrm{~mL}$ Fischer Porter reactor. The temperature of the reaction is set to $150^{\circ} \mathrm{C}$ and the solution was stirred for $21 \mathrm{~h}$. In the glovebox, $3 \mathrm{~mL}(0.096 \mathrm{mmol})$ as-synthesized $\mathrm{InP}$ QDs in mesitylene are added to a $100 \mathrm{~mL}$ round-bottom flask with $2 \mathrm{~mL}$ oleylamine (OLA). The temperature is set to $190^{\circ} \mathrm{C}$. $102.2 \mathrm{mg}$ of anhydrous $\mathrm{ZnCl}_{2}$ is added to a Schlenk tube with $2 \mathrm{~mL}$ OLA to yield a $0.66 \mathrm{M}$ solution which is warmed at $120^{\circ} \mathrm{C}$ to dissolve the solid. Additionally, a syringe with $0.13 \mathrm{~mL}(0.54 \mathrm{~mol})$ dodecanethiol (DDT) is prepared. At $190^{\circ} \mathrm{C}$,

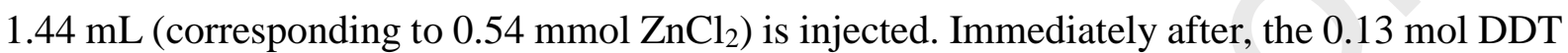
are injected, followed by an increase in temperature to $260^{\circ} \mathrm{C}$. The system is allowed to react for one hour during which time the solution turns from dark red to opaque brown and the reaction cooled to room temperature. To isolate the particles as a solid, the sample is purified with a 3:1 mixture of acetonitrile $(\mathrm{MeCN})$ and toluene. The product is centrifuged for 15 minutes at $20000 \mathrm{rpm}$, after which the non-colored supernatant is discarded. The sample is dried under vacuum for two hours. The final QD are therefore composed of InP/ZnS@OlA. Characterization of the NP is reported in the Supporting Information.

Elaboration of the composition maps. For each sample, water was added to the THF containing BHT and the NP (if present) in one shot, and the sample vortexed a few seconds. After ca 1 hour, the samples were visually inspected. For samples in the Ouzo domain, but close to the boundary with the monophasic domain, the haze was detected by observing the samples under the light of a telephone flash. A few samples exhibited a slight degree of creaming, but did not show an oily phase. These were located close to the boundary between the Ouzo and diphasic domains, and were attributed to the Ouzo domain. All experiments in which the amount of NP was varied were prepared at a fixed water/THF/BHT composition, using commercial THF containing a known amount of BHT (282 mg. $\mathrm{L}^{-1}$, determined using HPLC).

When mentioned, the NP-shells were crosslinked by adding $0.1 \mathrm{mg} \cdot \mathrm{mL}^{-1}$ of poly(acrylic acid) 450000 g.mole ${ }^{-1}$. Then the solvent was evaporated overnight in open vials placed at $40^{\circ} \mathrm{C}$ and the sample purified either by centrifugation (QD) of magnetic separation using a permanent magnet (SPION). 
Nanoparticle tracking analysis (NTA). Briefly, the principle of NTA is as follows. The dispersion is illuminated by a laser beam. The light scattered by the particles appears as individual spots that are recorded by a CCD camera through a microscope. The size of each object is then individually deduced from the analysis of its Brownian motion. In contrast to dynamic light scattering, particles of all sizes appear with the same weighting in the signal, which allows to obtain representative sample distributions, even for polydisperse samples. However, in extreme cases, very large or contrasting particles can mask the smaller ones. NTA was carried out with a Nanosight LM10 device system (Malvern Panalytical) equipped with a $40 \mathrm{~mW}$ laser working at $\lambda=638 \mathrm{~nm}$. Video sequences were recorded via a CCD camera operating at 30 frames per second and evaluated via the NANOSIGHT NTA 2.0 Analytical Software SuiteFor each sample, 3 acquisitions with an acquisition time of $60 \mathrm{~s}$ were performed at $25^{\circ} \mathrm{C}$. Calculations of the hydrodynamic diameters were achieved taking the viscosity of the water/THF mixture at the temperature of measurement from values reported by Mahendra et $\mathrm{al}^{33}$. Since NTA measurements are based on microscopic observation of the light spots scattered by the particles, the measurement of concentration is intrinsically limited to high concentrations (typically above $5 \times 10^{9}$ particles $/ \mathrm{mL}$ ) by the superposition of these light spots. It then no longer allows a precise determination of the concentration.

Dynamic Light Scattering (DLS) and zeta potential. DLS measurements were achieved using a Nanokin DLS device from Cordouan. To characterize the dynamics of droplet growth, the signal was acquired continuously during 20 minutes. The hydrodynamic diameter (Zaverage) is calculated by the methods of cumulants. The cumulant analysis of the autocorrelation signal was performed using sequences of 30 seconds every minute, thorough the duration of the experiment. Zeta potential was measured using a Malvern Nanosizer. Measurements were achieved in triplicates of 100 measurements each.

Inductively Coupled Plasma Atomic Emission Optical Spectroscopy (ICP-AES). The magnetic and non-magnetic fraction were digested in $500 \mu \mathrm{L}$ aqueous solution of HNO3 (68\%) in plastic vials. After one day, the total volume is adjusted with water to $25 \mathrm{~mL}$ and the samples titrated for Cd, Se and Fe using and ICP-EOS device (iCAP 7000Series, Thermo Scientific) under argon flux.

Electron transmission microscopy and Energy Dispersive X-ray Spectroscopy. The grids were prepared by deposition of a drop of the suspension to be analyzed on a copper grid (coated with either a single carbon film or with both carbon and formvar films). When using aqueous 
suspensions, the drop remained well formed (poor wetting conditions) and was let under the fume hood until totally evaporated. When using organic suspensions, the drop spread immediately over the grid (strong wetting). Transmission electron microscopy was performed using a JEOL JEM-2100 with a $\mathrm{LaB}_{6}$ source operating at $200 \mathrm{kV}$. The microscope is equipped with two cameras: a GATAN Orius SC200D and a Gatan UltraScan 1000XP HR. EDX spectra were acquired using an Oxford X-Max 80T detector.

1. Vitale, S. A. \& Katz, J. L. Liquid Droplet Dispersions Formed by Homogeneous Liquid-Liquid Nucleation: "The Ouzo Effect". Langmuir 19, 4105-4110 (2003).

2. Ganachaud, F., Aubry, J., Cohen Addad, J.-P. \& Cabane, B. Nanoprecipitation of Polymethylmethacrylate by Solvent Shifting:1. Boundaries. Langmuir 25, 1970-1979 (2009).

3. Ganachaud, F. et al. Modular construction of single-component polymer nanocapsules through a one-step surfactant-free microemulsion templated synthesis. Chem. Commun. 53, 1401-1404 (2017).

4. Chapel, J.-P., Pucci, C., Cousin, F., Dole, F. \& Schatz, C. Impact of the Formulation Pathway on the Colloidal State and Crystallinity of Poly- $\varepsilon$-caprolactone Particles Prepared by Solvent Displacement. Langmuir 34, 2531-2542 (2018).

5. Couvreur, P., Beck-Broichsitter, M. \& Nicolas, J. Solvent selection causes remarkable shifts of the "Ouzo region" for poly(lactide-co-glycolide) nanoparticles prepared by nanoprecipitation. Nanoscale 7, 9215-9221 (2015)

6. Couvreur, P., Lepeltier, E. \& Bourgaux, C. Nanoprecipitation and the "Ouzo effect": Application to drug delivery devices. Advanced Drug Delivery Reviews 71, 86-97 (2014).

7. Botet, R. \& Roger, K. How do interactions control droplet size during nanoprecipitation? Current Opinion in Colloid \& Interface Science 22, 108-112 (2016).

8. Lu, Z., Peng, S. \& Zhang, X. Influence of Solution Composition on the Formation of Surface Nanodroplets by Solvent Exchange. Langmuir 32, 1700-1706 (2016). 
9. Li, M., Bao, L., Yu, H. \& Zhang, X. Formation of Multicomponent Surface Nanodroplets by Solvent Exchange. J. Phys. Chem. C 122, 8647-8654 (2018).

10. Hou, W. \& Xu, J. Surfactant-free microemulsions. Current Opinion in Colloid \& Interface Science 25, 67-74 (2016).

11. Smith, G. D., Donelan, C. E. \& Barden, R. E. Oil-continuous microemulsions composed of hexane, water, and 2-propanol. Journal of Colloid and Interface Science 60, 488-496 (1977).

12. Subramanian, D., Klauda, J. B., Collings, P. J. \& Anisimov, M. A. Mesoscale Phenomena in Ternary Solutions of Tertiary Butyl Alcohol, Water, and Propylene Oxide. J. Phys. Chem. B 118, 5994-6006 (2014).

13. Sedlák, M. Large-Scale Supramolecular Structure in Solutions of Low Molar Mass Compounds and Mixtures of Liquids: I. Light Scattering Characterization. J. Phys. Chem. B 110, 4329-4338 (2006).

14. Sedlák, M. \& Rak, D. Large-Scale Inhomogeneities in Solutions of Low Molar Mass Compounds and Mixtures of Liquids: Supramolecular Structures or Nanobubbles? J. Phys. Chem. B 117, 2495-2504 (2013).

15. Sedlák, M. \& Rak, D. On the Origin of Mesoscale Structures in Aqueous Solutions of Tertiary Butyl Alcohol: The Mystery Resolved. J. Phys. Chem. B 118, 2726-2737 (2014).

16. Zemb, T. N. et al. How to explain microemulsions formed by solvent mixtures without conventional surfactants. Proc Natl Acad Sci USA 113, 4260-4265 (2016).

17. Bago Rodriguez, A. M. \& Binks, B. P. Capsules from Pickering emulsion templates. Current Opinion in Colloid \& Interface Science 44, 107-129 (2019).

18. Ganachaud, F. \& Katz, J. L. Nanoparticles and Nanocapsules Created Using the Ouzo Effect: Spontaneous Emulsification as an Alternative to Ultrasonic and High-Shear Devices. ChemPhysChem 6, 209-216 (2005).

19. Sciortino, F. et al. Simple Engineering of Polymer-Nanoparticle Hybrid Nanocapsules. ChemNanoMat 2, 796799 (2016).

20. Sciortino, F. et al. Structure and elasticity of composite nanoparticle/polymer nanoshells (hybridosomes ${ }^{\circledR}$ ). Soft Matter 13, 4393-4400 (2017).

21. Goubault, C. et al. The Ouzo effect: A tool to elaborate high-payload nanocapsules. Journal of Controlled Release (2020) doi:10.1016/j.jconrel.2020.05.023.

22. Jiang, H., Sheng, Y. \& Ngai, T. Pickering emulsions: Versatility of colloidal particles and recent applications. Current Opinion in Colloid \& Interface Science 49, 1-15 (2020). 
23. Gross, J., Sayle, S., Karow, A. R., Bakowsky, U. \& Garidel, P. Nanoparticle tracking analysis of particle size and concentration detection in suspensions of polymer and protein samples: Influence of experimental and data evaluation parameters. European Journal of Pharmaceutics and Biopharmaceutics 104, 30-41 (2016).

24. Filipe, V., Hawe, A. \& Jiskoot, W. Critical Evaluation of Nanoparticle Tracking Analysis (NTA) by NanoSight for the Measurement of Nanoparticles and Protein Aggregates. Pharm Res 27, 796-810 (2010).

25. Diat, O. et al. Octanol-rich and water-rich domains in dynamic equilibrium in the pre-ouzo region of ternary systems containing a hydrotrope. J Appl Crystallogr 46, 1665-1669 (2013).

26. Arditty, S., Whitby, C. P., Binks, B. P., Schmitt, V. \& Leal-Calderon, F. Some general features of limited coalescence in solid-stabilized emulsions. The European Physical Journal E 9.

27. Coppel, Y. et al. Self-Assembly of ZnO Nanoparticles - An NMR Spectroscopic Study. European Journal of Inorganic Chemistry 2012, 2691-2699 (2012).

28. Coppel, Y. et al. Full Characterization of Colloidal Solutions of Long-Alkyl-Chain-Amine-Stabilized ZnO Nanoparticles by NMR Spectroscopy: Surface State, Equilibria, and Affinity. Chemistry - A European Journal 18, 5384-5393 (2012).

29. Wiley, R. M. Limited coalescence of oil droplets in coarse oil-in-water emulsions. Journal of Colloid Science 9, 427-437 (1954).

30. Schmitt, V., Arditty, S. \& Leal-Calderon, F. Chapter 15 - Stability of concentrated emulsions. in Emulsions: Structure Stability and Interactions (ed. Petsev, D. N.) vol. 4 607-639 (Elsevier, 2004).

31. Binks, B. P. Particles as surfactants $\square$ similarities and differences. Interface Science 21 (2002).

32. Casterou, G. et al. Improved Transversal Relaxivity for Highly Crystalline Nanoparticles of Pure $\mathrm{y}^{-\mathrm{Fe}_{2} \mathrm{O}_{3}}$ Phase. Chem. Eur. J. 21, 18855-18861 (2015).

33. Mahendra, N. R., Das, B. \& Hazra, D. K. Densities and viscosities of the binary aqueous mixtures of tetrahydrofuran and 1,2-dimethoxyethane at 298, 308 and 318 K. INDIAN J. CHEM. TECHNOL. 5 (1994). 